\title{
Fast magnetoacoustic wave trains in coronal holes
}

\author{
D. J. Pascoe ${ }^{1}$, V. M. Nakariakov ${ }^{1,2,3}$, and E. G. Kupriyanova ${ }^{3}$
}

\author{
${ }^{1}$ Centre for Fusion, Space and Astrophysics, Department of Physics, University of Warwick, CV4 7AL, UK \\ e-mail: D.J.Pascoe@warwick.ac.uk \\ 2 School of Space Research, Kyung Hee University, Yongin, 446-701 Gyeonggi, Korea \\ 3 Central Astronomical Observatory at Pulkovo of the Russian Academy of Sciences, 196140 St Petersburg, Russia
}

Received 2 April 2014 / Accepted 26 June 2014

\begin{abstract}
Context. Rapidly propagating coronal EUV disturbances recently discovered in the solar corona are interpreted in terms of guided fast magnetoacoustic waves. Fast magnetoacoustic waves experience geometric dispersion in waveguides, which causes localised, impulsive perturbations to develop into quasi-periodic wave trains.

Aims. We consider the formation of fast wave trains in a super-radially expanding coronal hole modelled by a magnetic funnel with a field-aligned density profile that is rarefied in comparison to the surrounding plasma. This kind of structure is typical of coronal holes, and it forms a fast magnetoacoustic anti-waveguide as a local maximum in the Alfvén speed.

Methods. We performed 2D MHD numerical simulations for impulsively generated perturbations to the system. Both sausage and kink perturbations are considered and the role of the density contrast ratio investigated.

Results. The anti-waveguide funnel geometry refracts wave energy away from the structure. However, in this geometry the quasiperiodic fast wave trains are found to appear, too, and so can be associated with the observed rapidly propagating coronal EUV disturbances. The wave trains propagate along the external edge of the coronal hole. The fast wave trains generated in coronal holes exhibit less dispersive evolution than in the case of a dense waveguide.

Conclusions. We conclude that an impulsive energy release localised in a coronal plasma inhomogeneity develops into a fast wave train for both kink and sausage disturbances and for both waveguide and anti-waveguide transverse plasma profiles.
\end{abstract}

Key words. magnetohydrodynamics (MHD) - Sun: atmosphere - Sun: corona - Sun: magnetic fields - Sun: oscillations - waves

\section{Introduction}

The solar corona is a highly structured medium, which modifies the behaviour of magnetohydrodynamic (MHD) waves. The study of MHD wave interaction with coronal plasma nonuniformities is one of the hot topics of solar physics. The interest in waves is mainly motivated by the enigmatic problem of coronal heating, and by development of wave-based techniques of plasma diagnostics (see e.g. recent reviews by De Moortel \& Nakariakov 2012; Pascoe 2014). Observations of the solar corona with high time and spatial resolution revealed the occasional appearance of rapidly propagating quasi-periodic waves of the white light perturbations observed during eclipses (Williams et al. 2001, 2002; Katsiyannis et al. 2003) and, recently, of the EUV emission intensity (Liu et al. 2011, 2012). In both bands the rapidly propagating disturbances were interpreted as fast magnetoacoustic waves; see Cooper et al. (2003) for interpretating the white-light disturbances and Ofman et al. (2011) for the EUV disturbances. The rapidly propagating disturbances could also be responsible for the quasi-periodic variations in the polarised brightness in the polar coronal holes, detected by Ofman et al. (1997).

Confident detection of rapidly propagating coronal disturbances generated a number of follow-up studies in the EUV band. The typical apparent (projected) speeds of the disturbances were found to exceed several hundred $\mathrm{km} \mathrm{s}^{-1}$, and the periods are several tens of seconds (see e.g. Shen et al. 2013, for another recent event). Also, detailed analysis revealed the association of rapidly propagating EUV disturbances with impulsive energy releases (Shen \& Liu 2012) and the wave-train nature of the disturbances (Yuan et al. 2013). Formation of fast magnetoacoustic wave trains in field-aligned plasma waveguides is a well-known feature of impulsively generated dispersive waves, as pointed out in Roberts et al. (1983,1984). Numerical simulations carried out by Murawski \& Roberts (1993a,b,c, 1994) supported this suggestion. Nakariakov et al. (2004) demonstrated that a guided fast wave train has a characteristic "crazy tadpole" wavelet spectral signature. Those signatures have been found in wavelet spectra of rapidly propagating white-light disturbances (Katsiyannis et al. 2003; Nakariakov et al. 2004) and EUV disturbances (Yuan et al. 2013). Also, similar signatures are often detected in the integrated radio signals generated in solar flares (see Mészárosová et al. 2009a,b,c, 2011; Karlický et al. 2013). Moreover, similar evolution was numerically found in the interaction of magnetoacoustic waves with flare current sheets (Jelínek \& Karlický 2012; Jelínek et al. 2012) and with a fan structure above the magnetic null point in a standard model of a solar flare (Mészárosová et al. 2013).

An advanced model of the dispersive evolution and propagation of impulsively generated fast magnetoacoustic waves, which reproduces the observed behaviour of rapidly propagating coronal disturbances well, was recently developed by Pascoe et al. (2013) for an expanding magnetic funnel filled in with dense plasma. A field-aligned density enhancement forms a dispersive waveguide for fast magnetoacoustic waves. Additionally, the vertical stratification of the magnetic field causes the Alfvén 
speed outside the funnel to vary with height. The refraction of fast waves that leak out of the funnel provides a mechanism by which upwards-propagating quasi-periodic wave trains can be generated outside of the structure in which they were initially generated and evolved.

Another kind of structure common in the solar atmosphere is a coronal hole. These regions are defined by their open field lines and by a plasma that is less dense than the average surrounding values, and so they appear darker in EUV images. MHD wave activity has been widely observed in coronal holes (see e.g. reviews by Ofman 2005, 2009; Banerjee et al. 2011). From the fast magnetoacoustic wave dynamics, the main difference between a coronal loop or dense funnel and a coronal hole is the transverse profile of the plasma density. In the former structures, the density is enhanced. For a low- $\beta$ plasma, this corresponds to a decrease in the local Alfvén and fast magnetoacoustic speeds. Thus, coronal loops and dense funnels are fast magnetoacoustic waveguides (e.g. Edwin \& Roberts 1988; Nakariakov \& Roberts 1995). The locally-oblique fast waves experience refraction or reflection at the transverse non-uniformities and propagate along the field. In contrast, in coronal holes the transverse profile of the plasma density experiences a decrease that corresponds to an increase in the fast magnetoacoustic speed. Thus, coronal holes act as fast magnetoacoustic anti-waveguides (see e.g. Ofman \& Davila 1995). It is not known whether quasi-periodic fast wave trains can be formed in coronal anti-waveguides.

In this paper we consider the impulsive excitation of fast magnetoacoustic waves in a magnetic funnel with a field-aligned structure that describes a local deficit in the average plasma density. Such a configuration corresponds to a super-radially open coronal hole. This structure forms a local maximum in the Alfvén speed and so behaves as an anti-waveguide for fast magnetoacoustic waves. This model is therefore applicable to coronal holes and similar plasma configurations, such as cavities between two high density structures. The initial equilibrium for the model is described in Sect. 2. In Sect. 3 the results of numerical simulations are presented for two methods of exciting waves in the system: an antisymmetric velocity pulse (exciting sausage modes) and a symmetric velocity pulse (exciting kink modes). Further discussion of the results is presented in Sect. 4.

\section{Model setup}

Our equilibrium magnetic configuration is a $2 \mathrm{D}$ potential magnetic field as used in Pascoe et al. (2013)

$$
\boldsymbol{B}=B_{0} \exp (-\tilde{z})\left[\sin (\tilde{x}+\pi) \boldsymbol{e}_{x}+\cos (\tilde{x}+\pi) \boldsymbol{e}_{z}\right]
$$

where $B_{0}$ is a constant determining the magnitude of the magnetic field. The horizontal and vertical coordinates are denoted by $\tilde{x}$ and $\tilde{z}$, respectively, where a tilde denotes a dimensionless variable with a characteristic length $l_{0}$ being a normalising quantity. For this magnetic field, it is convenient to define the upper boundary of the numerical domain to be at $\tilde{z}=0$, and our numerical domain is defined for the ranges $\tilde{z}=[-\pi, 0]$ and $\tilde{x}=[-\pi, \pi]$ (e.g. Fig. 1).

The absolute value of the magnetic field decreases exponentially with height but is constant in the horizontal direction. The local variation of the Alfvén speed is therefore provided by the density profile, which is based on the general symmetric Epstein profile (e.g. Nakariakov \& Roberts 1995; Pascoe et al. 2007)

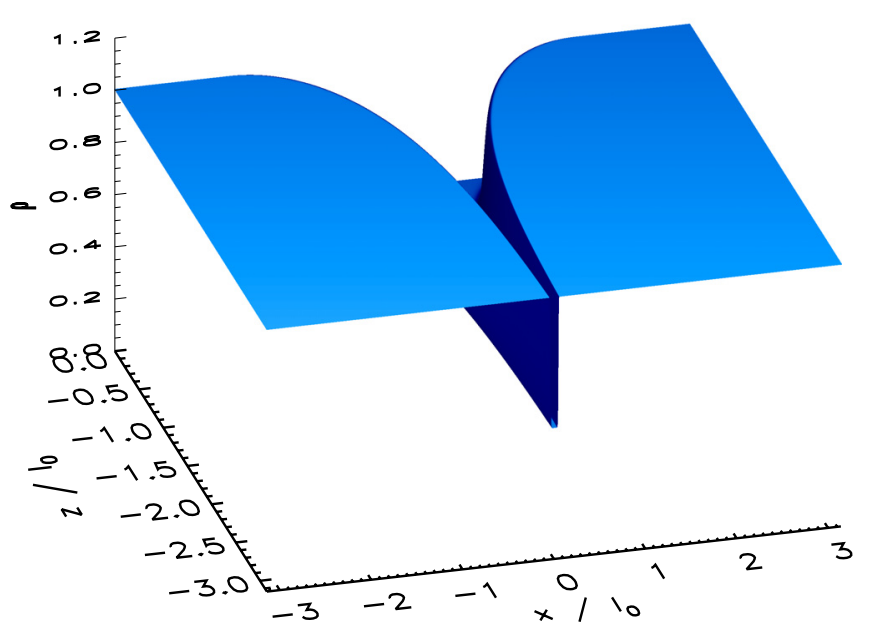

Fig. 1. Density profile for a funnel with density contrast $\rho_{\mathrm{F}} / \rho_{\infty}=-0.5$ and $p=8$. The profile follows the field lines of the potential magnetic field described by Eq. (1).

with a width that increases with height to remain aligned with the expanding magnetic field (Pascoe et al. 2013)

$\rho_{0}=\left(\rho_{\mathrm{F}}-\rho_{\infty}\right) \operatorname{sech}^{2}\left(\frac{\tilde{x}}{\operatorname{arccosexp}(\tilde{z})-\pi / 2}\right)^{p}+\rho_{\infty}$,

where $\rho_{\infty}$ is the (constant) density far from the funnel, $\rho_{\mathrm{F}}$ is the departure of the density in the centre of the funnel, and $p$ determines the profile steepness. The value of $\rho_{\mathrm{F}}$ can be either positive or negative so long as $\left|\rho_{\mathrm{F}}\right|<\rho_{\infty}$, and in this paper we consider the case of an anti-waveguide given by $\rho_{\mathrm{F}}<0$.

Figure 1 shows the density profile for a funnel with density contrast $\rho_{\mathrm{F}} / \rho_{\infty}=-0.5$ and $p=8$. The corresponding Alfvén speed profile is shown in Figure 2. The top panel shows the variation in the vertical direction (taken at the funnel axis $\tilde{x}=0$ ), which is proportional to the exponentially decreasing magnetic field strength. The bottom panel shows the variation in the horizontal direction (taken at $\tilde{z}=-0.2 \pi$ ), which forms an antiwaveguide (local maximum) due to the field-aligned density profile (Eq. (2) and Fig. 1).

The density structure is set in equilibrium by defining the internal energy density as

$\epsilon=\frac{P_{\mathrm{g}}}{\rho_{0}(\gamma-1)}$

where $P_{\mathrm{g}}$ is a constant gas pressure and $\gamma=5 / 3$ is the ratio of specific heat capacities. The plasma $\beta$ increases with height but remains small $(\beta<0.001)$ throughout the numerical domain. The low plasma $\beta$ means all temperatures are negligibly low and so excludes slow waves from our study.

Simulations were performed using the 2.5D MHD code Lare2d (Arber et al. 2001), which solves the ideal MHD equations by taking a Lagrangian predictor-corrector time step and remapping variables back onto the original Eulerian grid using van Leer gradient limiters. For our chosen coordinates, the $2.5 \mathrm{D}$ approximation corresponds to $\partial / \partial y=0$. Furthermore, $v_{y}$ and $B_{y}$ remain zero due to our choice of initial conditions, and so our model is essentially $2 \mathrm{D}$. The resolution used is $4000 \times 2000$ grid points, with convergence tests at a resolution of $8000 \times 4000$ grid points showing no significant differences. Line-tied boundary 

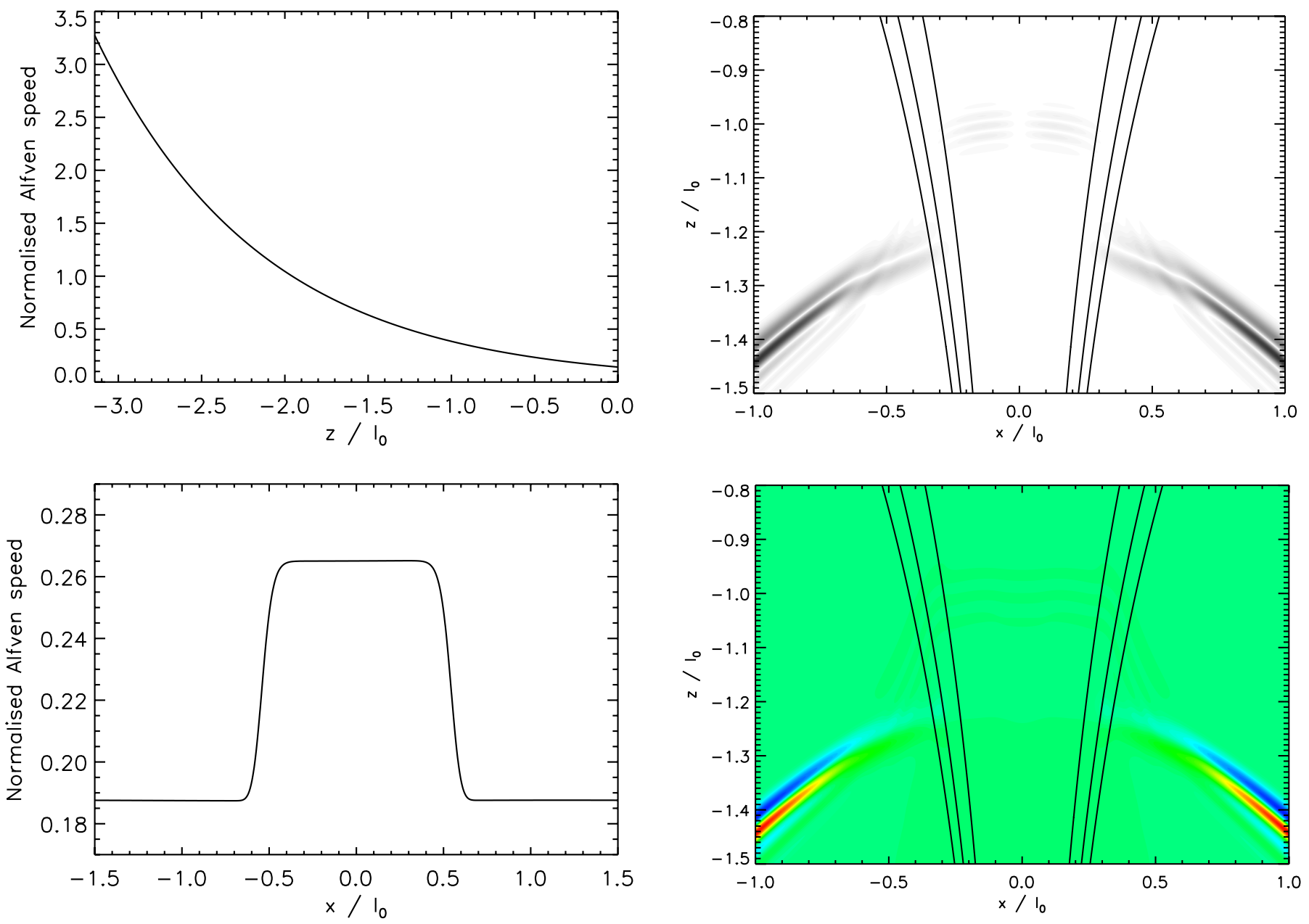

Fig. 2. Variation in the (normalised) Alfvén speed in the vertical direction (top; taken at $x=0$ ) and the horizontal direction (bottom; taken at $z=-0.2 \pi$ ). The magnetic field (Eq. (1)) provides vertical stratification while the density profile (Eq. (2) and Fig. 1) provides the local increase that defines the anti-waveguide structure.

conditions were used, with damping layers near the edges of the numerical domain to avoid perturbations reflecting back inwards. The domain has a size $2 \pi \times \pi$ in normalised units, which may be converted to physical units by choosing normalisation constants appropriate to the particular problem. The physical length scales $(x)$, time scales $(t)$, and speeds $(v)$ are related to the dimensionless variables (denoted by tildes) by $x=\tilde{x} l_{0}$, $t=\tilde{t} t_{0}$, and $v=\tilde{v} v_{0}$, where $l_{0}, t_{0}$, and $v_{0}$ are the chosen normalisation constants for length scales, times, and speeds, and $v_{0}=l_{0} / t_{0}$. For example, choosing $v_{0}=1 \mathrm{Mm} / \mathrm{s}, t_{0}=240 \mathrm{~s}$ (and so $l_{0}=240 \mathrm{Mm}$ ) means the Alfvén speeds in Fig. 2 represent the speed in $\mathrm{Mm} / \mathrm{s}$, the simulation runtime of $\tilde{t}=5$ corresponds to 20 minutes, and the numerical domain has a height of $\approx 750 \mathrm{Mm}$.

\section{Results}

\subsection{Sausage perturbations}

We excite fast waves by applying a perturbation inside the antiwaveguide structure near the bottom of the numerical domain. First we consider a spatially-localised compressive perturbation given by

$v_{x}=A x \exp \left[-\left(\frac{\tilde{x}-x_{0}}{\Delta_{x}}\right)^{2}\right] \exp \left[-\left(\frac{\tilde{z}-z_{0}}{\Delta_{z}}\right)^{2}\right]$,

Fig. 3. Snapshot of velocity $|\boldsymbol{v}|$ (top) and density (bottom) perturbations at $\tilde{t}=2$ for $\rho_{\mathrm{F}} / \rho_{\infty}=-0.5$ and $p=8$ and a sausage mode perturbation. The line contours outline the equilibrium density profile.

where $A$ is the initial amplitude, taken to be small enough to avoid nonlinear effects, and the parameters $\Delta_{x}$ and $\Delta_{z}$ are the width of the initial perturbation in the horizontal and vertical directions, respectively. The width parameters are also normalised to $l_{0}$. The perturbation is located at $\left(x_{0}, z_{0}\right)=(0,-0.75 \pi)$ with $\Delta_{x}=\Delta_{z}=0.05$ chosen to make the perturbation comparable in size to the funnel at the height at which it is applied. This leads to the efficient generation and dispersion of fast sausage oscillations.

Pascoe et al. (2013) describe the case of a magnetic funnel with a field-aligned density enhancement. In that case, the fast wave energy is separated into two distinct components. Trapped sausage waves propagate along the funnel axis, while leaky components form wave trains outside the funnel, which initially propagate horizontally, but then gradually turn upwards due to refraction from the non-uniform Alfvén speed. These external wave trains appear on both sides of the funnel and are called "wing" wave trains.

Figure 3 (top panel) shows a snapshot of the velocity $|\boldsymbol{v}|$ perturbations at a time $\tilde{t}=2$ for a numerical simulation with $\rho_{\mathrm{F}} / \rho_{\infty}=-0.5$. The line contours outline the equilibrium density profile and indicate the transition from the low density internal region to the higher density environment. The figure is cropped to allow comparison of the internal wave train (around $\tilde{z}=-1$ ) with the wing wave trains entering the frame in the bottom left- and right-hand corners. The internal wave train 


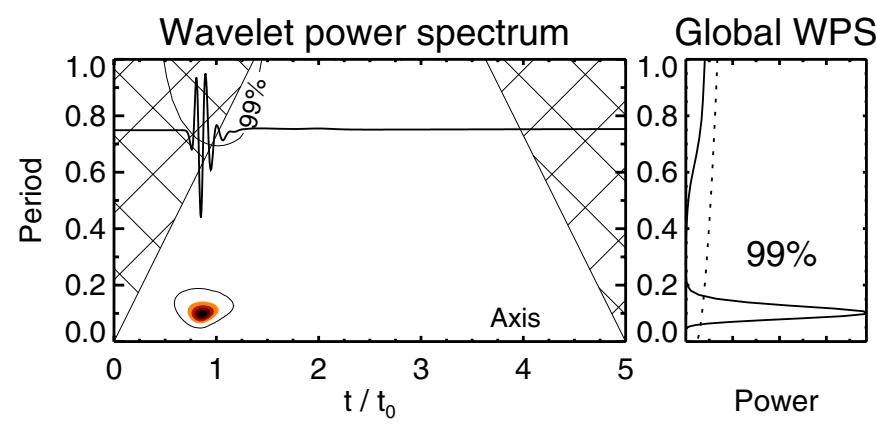

Fig. 4. Wavelet analysis of the density signal (inset curve) inside a funnel with $\rho_{\mathrm{F}} / \rho_{\infty}=-0.5$.

propagates with a faster speed in accordance with the higher local Alfvén speeds there (Fig. 2).

The quasi-periodic nature of the wave train is clearly seen in the snapshots of the generated signal. We illustrate it by the wavelet analysis of the density perturbations (measured at $\tilde{x}=0$ and $\tilde{z}=-\pi / 2$ ) in Fig. 4. Here and in subsequent wavelets the colour panel represents the Morlet wavelet power spectrum of the signal. The colour contours correspond to levels of wavelet power higher than $0.2,0.4,0.6$, and 0.8 times the maximum value. The solid contour shows the $99 \%$ significance level given by Torrence \& Compo (1998). The normalised time profile of the signal is superimposed on the wavelet spectrum as a solid line. The cross-hatched regions on either end of the wavelet spectrum indicate the cone of influence. The panel to the right of the wavelet spectrum represents the global wavelet power spectrum (WPS) of the signal with the dashed line defining the $99 \%$ significance level.

The wave energy associated with the oscillations inside the anti-waveguide is only a small fraction of the energy in the wing wave trains, so the corresponding density perturbations (bottom panel of Fig. 3) have negligibly low amplitudes. The same is true for the case of a kink perturbation (Sect. 3.2), so we focus our attention on the behaviour of the wing wave trains.

Figure 5 shows the velocity and density perturbations at a later time of $\tilde{t}=5$. The quasi-periodic wing wave trains are clearly visible on both sides of the anti-waveguide. The density perturbation signal passing a fixed point at $(\tilde{x}, \tilde{z})=$ $(-\pi / 2,-\pi / 2)$ and its wavelet power are shown in Fig. 6. Fewer periods of oscillation are seen (i.e. a lower oscillation quality) than the case of the waveguide model considered in Pascoe et al. (2013).

Figures 7 and 8 show the density perturbation signals for funnel anti-waveguides with $\rho_{\mathrm{F}} / \rho_{\infty}=-0.9$ and -0.1 , respectively. It can be seen that the signals weakly depend on the antiwaveguide density contrast over the full range of permitted values $-1<\rho_{\mathrm{F}} / \rho_{\infty}<0$.

\subsection{Kink perturbations}

Next we consider the case of a kink perturbation, i.e. a transverse displacement of the funnel axis. The applied perturbation has the form

$v_{x}=A \exp \left[-\left(\frac{\tilde{x}-x_{0}}{\Delta_{x}}\right)^{2}\right] \exp \left[-\left(\frac{\tilde{z}-z_{0}}{\Delta_{z}}\right)^{2}\right]$

where all variables have the same definition as in Eq. (4).
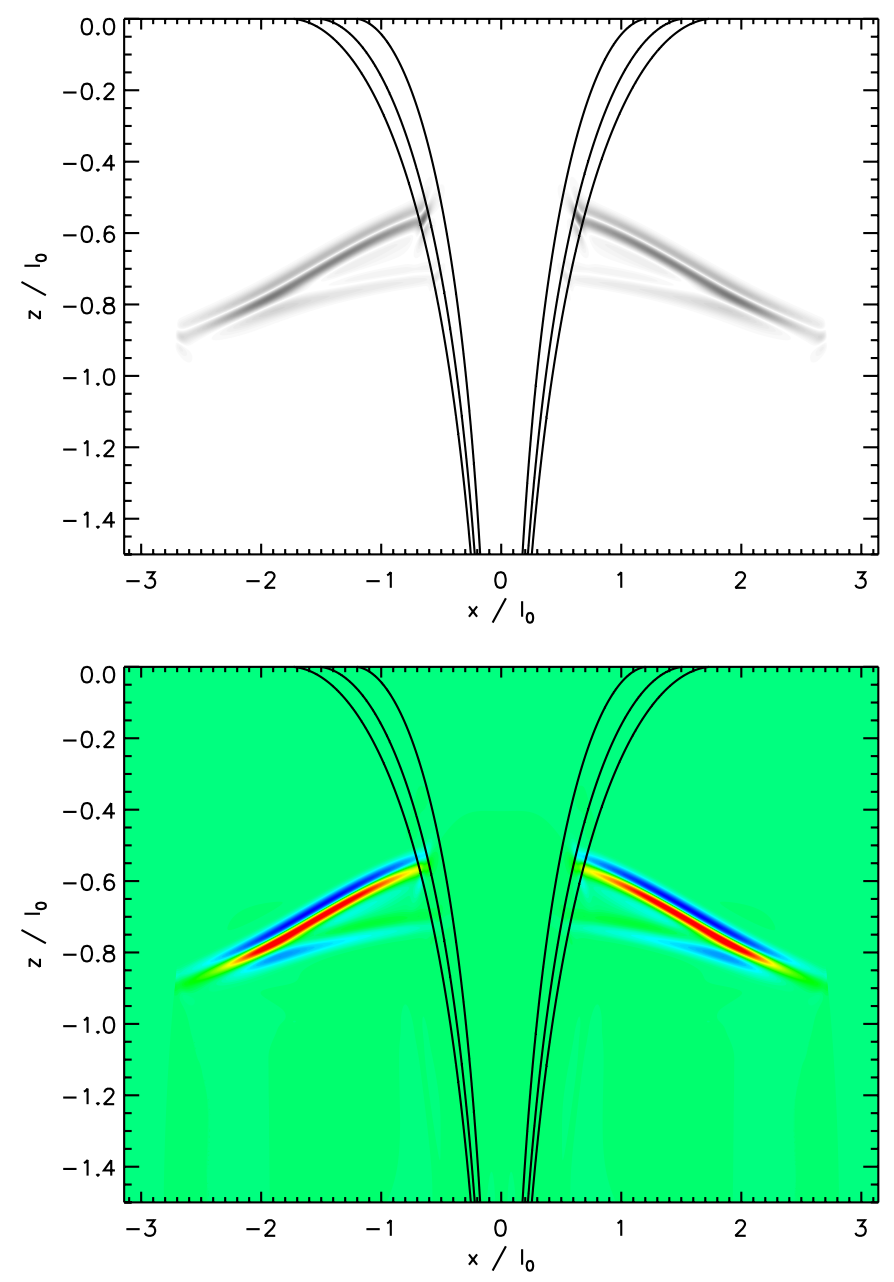

Fig. 5. Snapshot of velocity $|\boldsymbol{v}|$ (top) and density (bottom) perturbations at $\tilde{t}=5$ for $\rho_{\mathrm{F}} / \rho_{\infty}=-0.5$ and $p=8$ and a sausage mode perturbation. The line contours outline the equilibrium density profile.

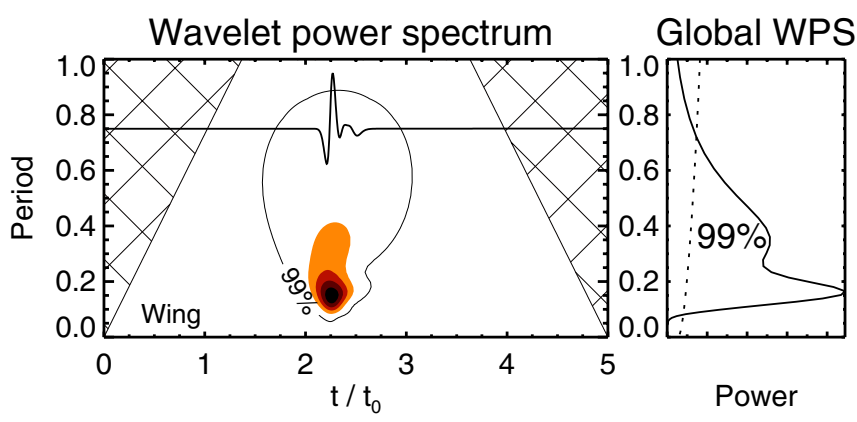

Fig. 6. Wavelet analysis of the density signal (inset curve) outside a funnel with $\rho_{\mathrm{F}} / \rho_{\infty}=-0.5$.

Figure 9 shows the density perturbations at a time of $\tilde{t}=$ 5 for the kink perturbation. This driver also generates quasiperiodic wing wave trains similar to the case of the sausage perturbation (compare with Fig. 5). However, the density perturbations on each side of the anti-waveguide are now anti-symmetric in accordance with the kink mode driver exciting a transverse displacement. The case of kink perturbations in a dense funnel is considered in Appendix A.

\section{Discussion}

In this paper we have considered the behaviour of impulsively generated fast magnetoacoustic waves in a super-radially 


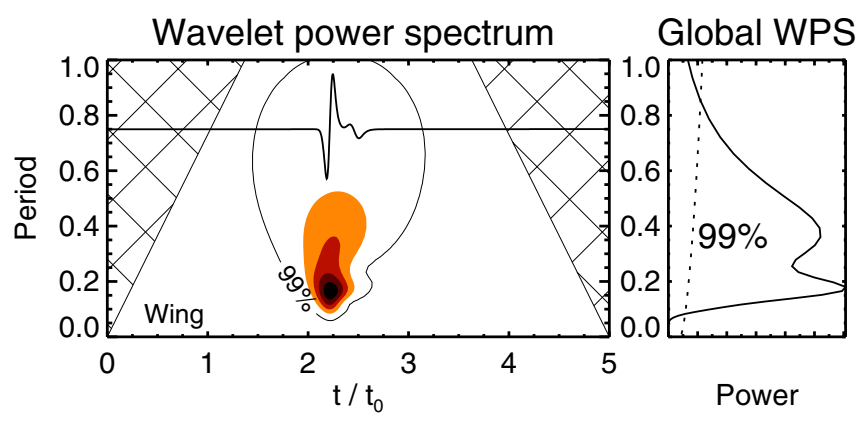

Fig. 7. Wavelet analysis of the density signal (inset curve) outside a funnel with $\rho_{\mathrm{F}} / \rho_{\infty}=-0.9$.

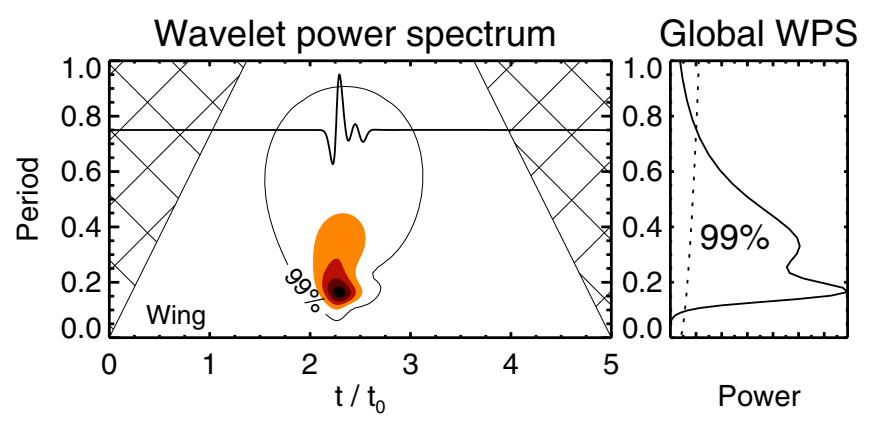

Fig. 8. Wavelet analysis of the density signal (inset curve) outside a funnel with $\rho_{\mathrm{F}} / \rho_{\infty}=-0.1$.

expanding coronal hole, modelled as an expanding magnetic flux slab filled in with a low- $\beta$ plasma, surrounded by a denser low- $\beta$ plasma. Such a plasma configuration acts as a fast magnetoacoustic anti-waveguide, because it has a bump of the fast magnetoacoustic speed. We considered fast magnetoacoustic waves excited by an impulsive source of the plasma motion spatially localised inside the hole. The main result of this study is that in the coronal hole plasma configuration, as well as in the previously considered case of a dense plasma funnel, fast waves develop in quasi-periodic wave trains. These wave trains resemble rapidly propagating quasi-periodic EUV disturbances recently discovered with SDO/AIA in the solar corona. Quasi-periodic wave trains are shown to form in both cases of the sausage and kink symmetries of the initial impulsive excitation. We conclude that the dispersively formed fast wave trains can be responsible for the rapidly propagating quasi-periodic EUV disturbances observed in coronal holes.

In agreement with the case of a dense coronal funnel, considered in Pascoe et al. (2013), fast wave trains appear inside the waveguide and outside it. In both cases, the external fast wave trains are subject to refraction that turns the local wave vector in the vertical direction. In contrast to the dense funnel case, in a coronal hole, external wave trains are much more pronounced than the internal wave trains, which is consistent with the antiwaveguide nature of the hole. Indeed, the fast waves cannot be trapped inside it and tend to "slide down" outside the hole in the horizontal direction. The quasi-periodic wave trains appearing in the coronal hole geometry are found to generally have a lower signal quality and are less sensitive to the density contrast ratio than the case of a dense funnel structure.

Our results obtained for the internal source of the fast waves are different from the case of waves excited outside the hole. Externally excited fast waves are observed to reflect away from coronal holes (e.g. Thompson et al. 1999) so cannot evolve in

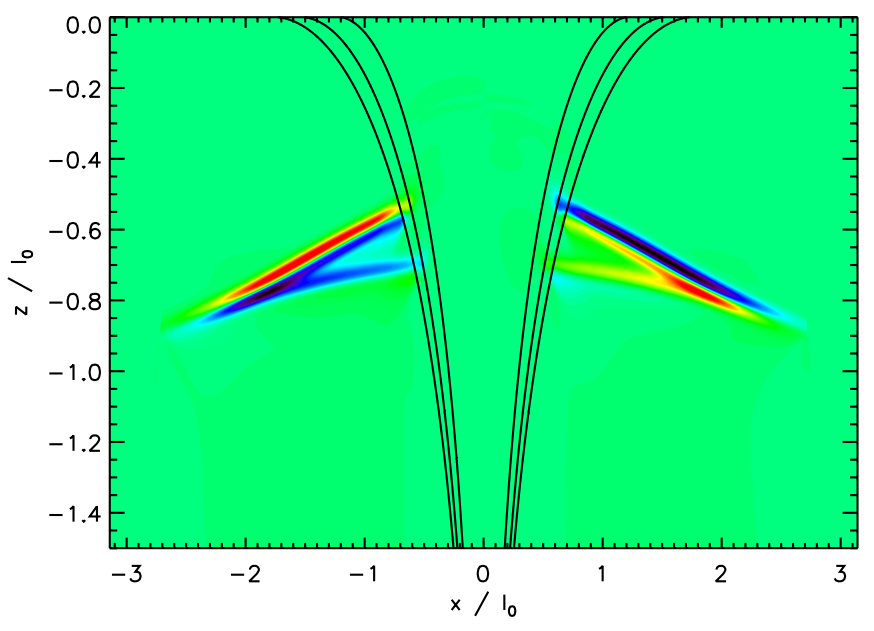

Fig. 9. Snapshot of density perturbations at $\tilde{t}=5$ for $\rho_{\mathrm{F}} / \rho_{\infty}=-0.5$ and $p=8$ and a kink mode perturbation. The line contours outline the equilibrium density profile.

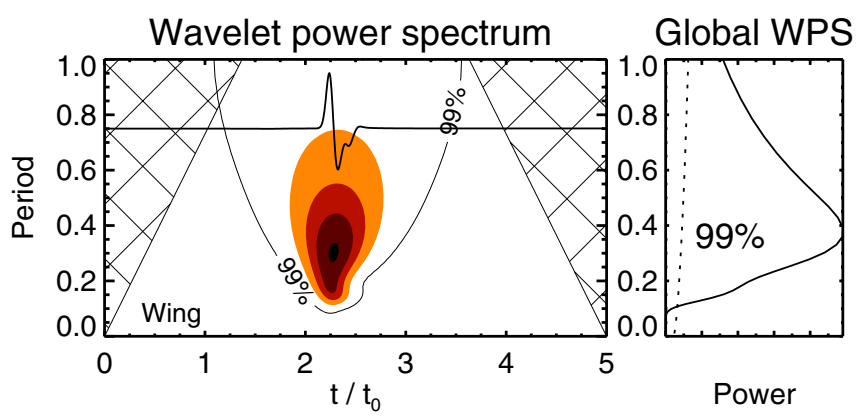

Fig. 10. Wavelet analysis of the density signal (inset curve) outside a funnel with $\rho_{\mathrm{F}} / \rho_{\infty}=-0.5$ in response to a kink perturbation.

quasi-periodic wave trains. Thus, the source of the rapidly propagating quasi-periodic EUV disturbances in coronal holes should be localised within the magnetic flux tube forming the hole.

In Appendix A we consider the evolution of kink perturbations in a dense plasma funnel. We found that, similar to the case of a sausage excitation, the initial perturbation evolves in internal and external quasi-periodic fast wave trains. Thus, fast wave trains are a robust feature of impulsive spatially localised energy releases in plasma inhomogeneities of the solar corona. Fast wave trains appear in both the cases of kink and sausage initial perturbations in both fast waveguides and anti-waveguides. For the case of a cylindrical geometry, the kink wave train propagating along the axis may also be subject to mode coupling (e.g. Pascoe et al. 2010; Hood et al. 2013), which will cause the wave train to undergo damping with a frequency-dependent rate that acts like a low-pass filter (Terradas et al. 2010). Since the coupling also applies to wave energy just outside the density structure (e.g. Fig. 9 of Pascoe et al. 2010), this may also affect the external wave train near to the funnel but not the parts farther away from the structure.

Acknowledgements. This work is supported by the Marie Curie PIRSES-GA2011-295272 RadioSun project, the European Research Council under the SeismoSun Research Project No. 321141 (DJP, VMN), the BK21 plus programme through the National Research Foundation funded by the Ministry of Education of Korea and the Russian Foundation of Basic Research grant 13-02-00044 (VMN). The computational work for this paper was carried out on the joint STFC and SFC (SRIF) funded cluster at the University of St Andrews (Scotland, UK). Wavelet software was provided by C. Torrence and G. Compo, and is available at URL: http://atoc.colorado.edu/ research/wavelets/ 
Appendix A: Kink perturbations for a dense funnel
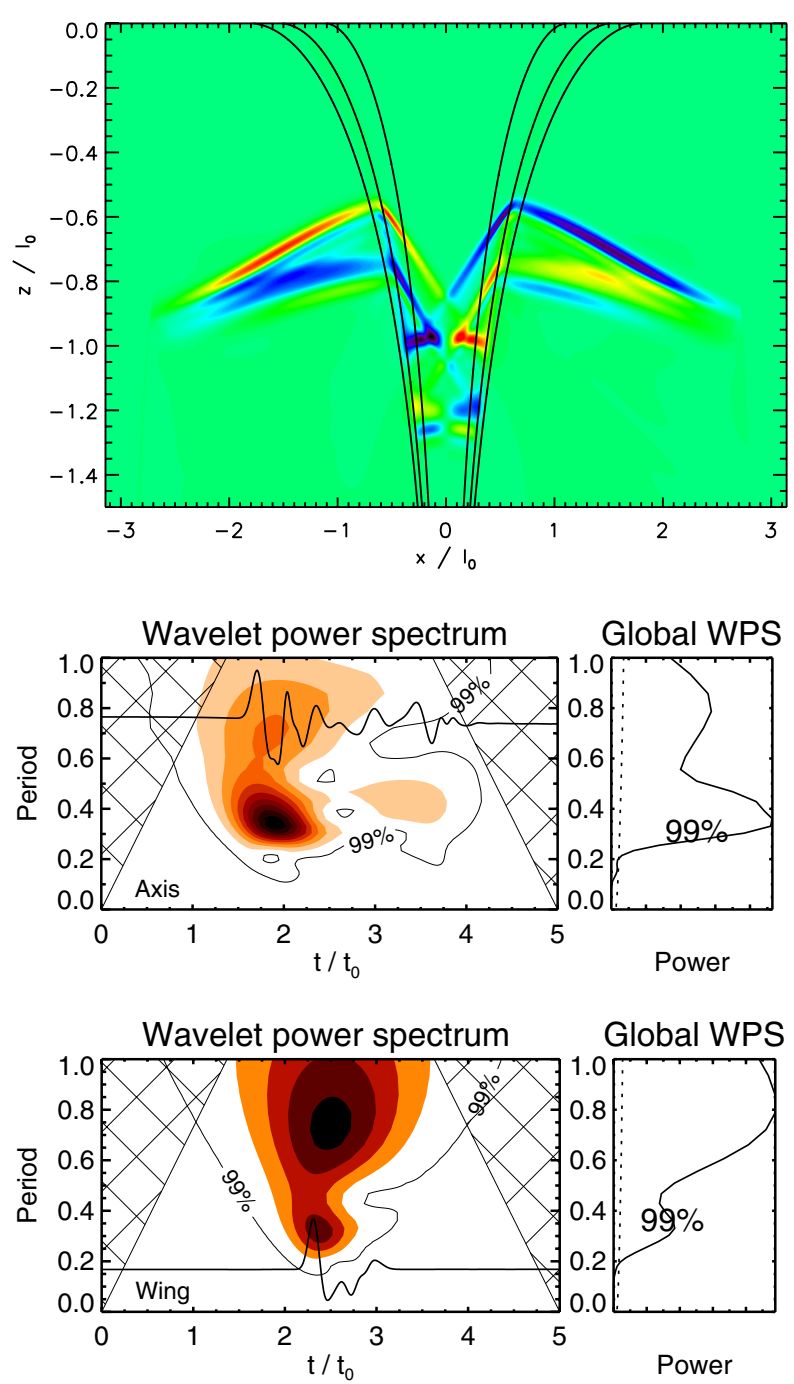

Fig. A.1. Snapshot of density perturbations (top) at $\tilde{t}=5$ for a dense funnel with $\rho_{\mathrm{F}} / \rho_{\infty}=3$ and $p=8$ and a kink mode perturbation. The line contours outline the equilibrium density profile. The middle and bottom panels show the wavelet analysis for the density perturbations measured near the axis and in the wing, respectively.

To allow further comparison of the results in Sect. 3.2 with the case of a dense coronal funnel studied by Pascoe et al. (2013), we return here to the model in that paper for the case of a symmetric velocity perturbation given by Eq. (5). Figure A.1 shows the results for a funnel with density contrast $\rho_{\mathrm{F}} / \rho_{\infty}=3$ and $p=8$. The top panel shows a snapshot of the density perturbations at $\tilde{t}=5$. The middle and bottom panels show the time signatures for the density perturbations measured near the axis and in the wing, respectively. Quasi-periodic wave trains are seen both inside and outside the funnel, and the dispersive evolution is greater than the case of a rarefied anti-waveguide.

\section{References}

Arber, T., Longbottom, A., Gerrard, C., \& Milne, A. 2001, J. Comput. Phys., 171,151

Banerjee, D., Gupta, G. R., \& Teriaca, L. 2011, Space Sci. Rev., 158, 267

Cooper, F. C., Nakariakov, V. M., \& Williams, D. R. 2003, A\&A, 409, 325

De Moortel, I., \& Nakariakov, V. M. 2012, Roy. Soc. London Philos. Trans. Ser. A, 370, 3193

Edwin, P. M., \& Roberts, B. 1988, A\&A, 192, 343

Hood, A. W., Ruderman, M., Pascoe, D. J., et al. 2013, A\&A, 551, A39

Jelínek, P., \& Karlický, M. 2012, A\&A, 537, A46

Jelínek, P., Karlický, M., \& Murawski, K. 2012, A\&A, 546, A49

Karlický, M., Mészárosová, H., \& Jelínek, P. 2013, A\&A, 550, A1

Katsiyannis, A. C., Williams, D. R., McAteer, R. T. J., et al. 2003, A\&A, 406, 709

Liu, W., Title, A. M., Zhao, J., et al. 2011, ApJ, 736, L13

Liu, W., Ofman, L., Nitta, N. V., et al. 2012, ApJ, 753, 52

Mészárosová, H., Karlický, M., Rybák, J., \& Jiřička, K. 2009a, A\&A, 502, L13

Mészárosová, H., Karlický, M., Rybák, J., \& Jiřička, K. 2009b, ApJ, 697, L108

Mészárosová, H., Sawant, H. S., Cecatto, J. R., et al. 2009c, Adv. Space Res., 43, 1479

Mészárosová, H., Karlický, M., \& Rybák, J. 2011, Sol. Phys., 273, 393

Mészárosová, H., Dudík, J., Karlický, M., Madsen, F. R. H., \& Sawant, H. S. 2013, Sol. Phys., 283, 473

Murawski, K., \& Roberts, B. 1993a, Sol. Phys., 143, 89

Murawski, K., \& Roberts, B. 1993b, Sol. Phys., 144, 101

Murawski, K., \& Roberts, B. 1993c, Sol. Phys., 144, 255

Murawski, K., \& Roberts, B. 1994, Sol. Phys., 151, 305

Nakariakov, V. M., \& Roberts, B. 1995, Sol. Phys., 159, 399

Nakariakov, V. M., Arber, T. D., Ault, C. E., et al. 2004, MNRAS, 349, 705

Ofman, L. 2005, Space Sci. Rev., 120, 67

Ofman, L. 2009, Space Sci. Rev., 149, 153

Ofman, L., \& Davila, J. M. 1995, J. Geophys. Res., 100, 23413

Ofman, L., Romoli, M., Poletto, G., Noci, G., \& Kohl, J. L. 1997, ApJ, 491, L111

Ofman, L., Liu, W., Title, A., \& Aschwanden, M. 2011, ApJ, 740, L33

Pascoe, D. J. 2014, Res. Astron. Astrophys., 14, 805

Pascoe, D. J., Nakariakov, V. M., \& Arber, T. D. 2007, A\&A, 461, 1149

Pascoe, D. J., Wright, A. N., \& De Moortel, I. 2010, ApJ, 711, 990

Pascoe, D. J., Nakariakov, V. M., \& Kupriyanova, E. G. 2013, A\&A, 560, A97

Roberts, B., Edwin, P. M., \& Benz, A. O. 1983, Nature, 305, 688

Roberts, B., Edwin, P. M., \& Benz, A. O. 1984, ApJ, 279, 857

Shen, Y., \& Liu, Y. 2012, ApJ, 753, 53

Shen, Y.-D., Liu, Y., Su, J.-T., et al. 2013, Sol. Phys., 288, 585

Terradas, J., Goossens, M., \& Verth, G. 2010, A\&A, 524, A23

Thompson, B. J., Gurman, J. B., Neupert, W. M., et al. 1999, ApJ, 517, L151

Torrence, C., \& Compo, G. P. 1998, Bulletin of the American Meteorological Society, 79, 61

Williams, D. R., Phillips, K. J. H., Rudawy, P., et al. 2001, MNRAS, 326, 428

Williams, D. R., Mathioudakis, M., Gallagher, P. T., et al. 2002, MNRAS, 336, 747

Yuan, D., Shen, Y., Liu, Y., et al. 2013, A\&A, 554, A144 